\title{
Algorithm for Numerical Solution of Diffraction Problem on the Joint of Two Open Three-Layer Waveguides
}

\author{
Dmitriy Divakov ${ }^{1}$, Anastasiia Tiutiunnik ${ }^{1}$, and Anton Sevastianov ${ }^{1}$ \\ ${ }^{1}$ Peoples' Friendship University of Russia (RUDN University), 6 Miklukho-Maklaya St, Moscow, 117198, Russian Federation
}

\begin{abstract}
This paper describes the algorithm for the numerical solution of the diffraction problem of waveguide modes at the joint of two open planar waveguides. For planar structures under consideration, we can formulate a scalar diffraction problem, which is a boundary value problem for the Helmholtz equation with a variable coefficient in two-dimensional space. The eigenmode problem for an open three-layer waveguide is the Sturm-Liouville problem for a second-order operator with piecewise constant potential on the axis, where the potential is proportional to the refractive index. The described problem is singular and has a mixed spectrum and therefore the Galerkin method can not be used in this definition. One way to adapt the Galerkin method for the problem solution is to artificially limit the area, which is equivalent to placing the open waveguide in question in a hollow closed waveguide whose boundaries are remote from the real boundaries of the waveguide layer of the open waveguide. Thus, we obtain a diffraction problem on a finite interval and with a discrete spectrum, which can be solved by the projection method, as done in this paper.
\end{abstract}

\section{Introduction}

The paper presents the definition of the diffraction problem for waveguide modes at the joint of two open planar three-layer waveguides. The waveguide joint is the simplest model of waveguide transition, and therefore the description of the diffraction problem at the interface is similar to the definition of the problem for a waveguide transition [1-3]. The classical method for solving diffraction problems in closed waveguides is the so-called incomplete Galerkin method [4-7]. The incomplete Galerkin method is a projection method for solving waveguide problems, which uses the Kantorovich decomposition to represent the approximate solution [8]. For description of the problem, Sveshnikov's partial radiation conditions are used $[9,10]$.

The propagation of radiation in open waveguides is described by Maxwell's equations, boundary conditions, material equations, and asymptotic conditions.

The Maxwell's equations and the material equations in the Gaussian system of units have the following form $[1-3,11]$ :

$$
\begin{gathered}
\operatorname{rot} \vec{E}=-\frac{1}{c} \frac{\partial \vec{B}}{\partial t}, \operatorname{rot} \vec{H}=\frac{1}{c} \frac{\partial \vec{D}}{\partial t}, \\
\vec{D}=\varepsilon \vec{E}, \vec{B}=\mu \vec{H},
\end{gathered}
$$

where $\vec{E}, \vec{H}$ are vectors of electric and magnetic field intensities; $\vec{D}, \vec{B}$ are vectors of electric and magnetic inductions; $c$ is speed of light in vacuum; $\varepsilon$ is dielectric permittivity, and $\mu$ is magnetic permeability of the medium.
The boundary conditions on the boundaries of dielectric waveguide layers, imply the continuity of the tangential components of the electromagnetic field [1-3]:

$$
\left[\vec{E}^{\tau}\right]_{x=0, h_{\alpha}}=\left[\vec{H}^{\tau}\right]_{x=0, h_{\alpha}}=0
$$

where $[f]_{s=a}=f(a-0)-f(a+0)$ is a jump of the function $f$ at the point $s=a$, and tangential components of the electromagnetic field $\vec{E}^{\tau}$ and $\vec{H}^{\tau}$ are defined as $\vec{E}^{\tau}=\vec{E} \times \vec{n}, \vec{H}^{\tau}=\vec{H} \times \vec{n}$, where $\vec{n}$ is a normal to the layers interface.

The asymptotic conditions at an infinite distance from the upper boundary of the waveguide layer and, respectively, the lower boundary, are described assuming the field are bounded [1-3]:

$$
|\vec{E}|_{x \rightarrow \pm \infty} \leq C_{E},|\vec{H}|_{x \rightarrow \pm \infty} \leq C_{H}
$$

where $C_{E}$ and $C_{H}$ are some non-negative constants, and for guided waveguide modes $C_{E}=C_{H}=0$.

\section{Problem definition for TE-modes}

We will consider open three-layer waveguides of different cross-section and with different refractive indices of the waveguide layers. Waveguides are joined at $z=0$. Each of the three-layer waveguides occupies the entire space, the waveguide layer of the left waveguide is characterized by thickness $h_{1}$ and refractive index $n_{f}^{(1)}$, right one is described by $h_{2}$ and 
$n_{f}^{(2)}$. Covering layer $\left(x>h_{1,2}\right)$ is described by refractive index $n_{c}$. The substrate $(x<0)$ is described by refractive index $n_{s}$ and for both waveguides has semiinfinite thickness. The structure described is illustrated on Fig. 1.

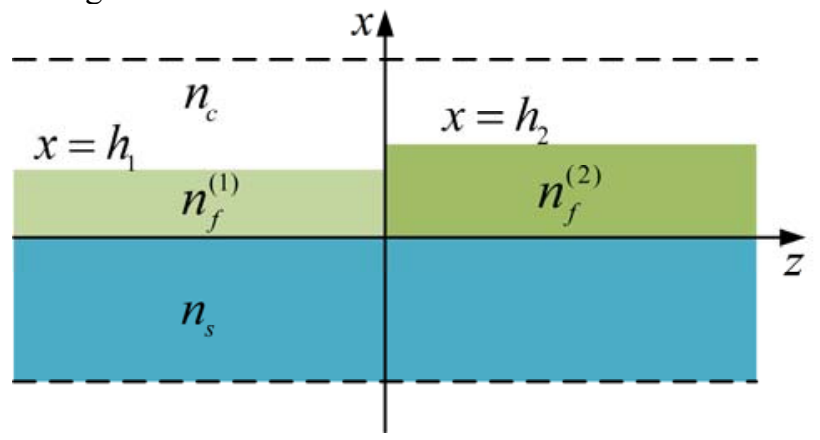

Fig. 1. Structure of two waveguides joint.

Refractive index $n(x, z)$ of the illustrated structure is defined as follows:

$$
n_{1}(x)=\left\{\begin{array}{c}
n_{c}, \quad x>h_{1} \\
n_{f}^{(1)}, 0<x<h_{1}, n_{2}(x)=\left\{\begin{array}{c}
n_{c}, \quad x>h_{2} \\
n_{s}, \quad x<0
\end{array} n_{f}^{(2)}, 0<x<h_{2} .\right. \\
n_{s}, \quad x<0
\end{array}\right.
$$

where $\left.n(x, z)\right|_{z<0}=n_{1}(x)$ and $\left.n(x, z)\right|_{z>0}=n_{2}(x)$.

The propagation of radiation in open waveguides is described by Maxwell's equations, boundary conditions, and material equations [1-3]. The geometry of planar structures does not change along the axis $O y$ and for time-harmonic fields (that do not change along axis $O y$ as well) Maxwell's equations are reduced to two independent subsystems - a subsystem for TE-modes and a subsystem for TM-modes [1-3]. Each subsystem can be described by a single Helmholtz equation with respect to the leading component of the field and two additional relations [1-3]. Therefore, for the planar structures under consideration, we can describe scalar diffraction problem, which is a boundary value problem for the Helmholtz equation with a variable coefficient in two-dimensional space.

The components of time-harmonic fields depend on time as $e^{-i \omega t}$. For the fields that are constant along $O y$, the relations $\partial E_{\alpha} / \partial y=0$ and $\partial H_{\alpha} / \partial y=0$ where $\alpha=x, y, z$, are fulfilled. With the assumptions described above, Maxwell's equations are represented as a subsystem for TE-modes [1-6, 9, 12]:

$$
\begin{aligned}
& \frac{\partial H_{x}}{\partial z}-\frac{\partial H_{z}}{\partial x}=-i k_{0} \varepsilon E_{y}, \\
& H_{x}=-\frac{1}{i k_{0} \mu} \frac{\partial E_{y}}{\partial z}, H_{z}=\frac{1}{i k_{0} \mu} \frac{\partial E_{y}}{\partial x}
\end{aligned}
$$

and a second subsystem linking the components of the TM-modes:

$$
\begin{aligned}
& \frac{\partial E_{x}}{\partial z}-\frac{\partial E_{z}}{\partial x}=i k_{0} \mu H_{y}, \\
& E_{x}=\frac{1}{i k_{0} \varepsilon} \frac{\partial H_{y}}{\partial z}, E_{z}=-\frac{1}{i k_{0} \varepsilon} \frac{\partial H_{y}}{\partial x}
\end{aligned}
$$

where $k_{0}$ is wave number in vacuum, $\varepsilon$ is dielectric permittivity, and $\mu$ is magnetic permeability of the waveguide. The subsystem for TE-modes can be represented as the Helmholtz equation with respect to the leading component $E_{y}$ and two relations describing $H_{x}$ and $H_{z}$ by the leading component. Thus, the diffraction problem for a planar structure can be defined as a scalar problem - a boundary value problem for the Helmholtz equation.

We will consider only the TE-mode, the description of the problem for the TM-mode is constructed in a similar way. For the planar structures under consideration, we can formulate a scalar diffraction problem, which is a boundary value problem for the Helmholtz equation with a variable coefficient in twodimensional space. The subsystem for the TE-mode can be formulated as the Helmholtz equation $[1-6,9,12]$ :

$$
\left(\frac{\partial^{2}}{\partial x^{2}}+\frac{\partial^{2}}{\partial z^{2}}+k_{0}^{2} n^{2}(x, z)\right) E_{y}=0
$$

where $n^{2}(x, z)$ is the refractive index of the waveguide under consideration, and two additional relations

$$
H_{x}=-\frac{1}{i k_{0} \mu} \frac{\partial E_{y}}{\partial z}, H_{z}=\frac{1}{i k_{0} \mu} \frac{\partial E_{y}}{\partial x} .
$$

The left and right waveguides joining at $z=0$, are three-layer regular waveguides, that is, for the left and right waveguides, the refractive indices in the equation are different functions, depending only on $x$. That's why we can split the variables for the left and right waveguides. At $z=0$ the boundary conditions of the continuity of the tangential components of the field are applied. The main difficulty in the study of diffraction problems in open waveguides is the existence of a continuous spectrum for the Sturm-Liouville problem for the transverse part $E_{y}$ after applying the method of separation of variables. In this paper, an artificial limitation of the area is used, which is equivalent to placing the open waveguide under consideration in a hollow closed waveguide which boundaries are remote from the real boundaries of the waveguide layer of the open waveguide. That is, we consider the problem not on the axis $-\infty<x<\infty$ but on the segment $-R \leq x \leq R$, where $R$ is several times greater than the thickness of the waveguide layer. The Dirichlet conditions $\left.E_{y}\right|_{x= \pm R}=0$ are fulfilled on borders $x= \pm R$. In the described method the continuous spectrum of the initial problem on the axis is roughly approximated by the discrete spectrum of the problem on the interval. The obtained approximate problem with a purely discrete spectrum can be solved using the Galerkin method. The described approach are called "the Dirichlet box".

By applying the separation of variables method to the Helmholtz equation (8) for the right waveguide and for 
the left waveguide, we can obtain a solution in the corresponding waveguide as a superposition of modes $C_{j}^{(\alpha)} \varphi_{j}^{(\alpha)}(x) e^{ \pm i k_{0} \beta_{j}^{(\alpha)} z}$, where $C_{j}^{(\alpha)}$ determines the amplitude of the corresponding mode, the sign in the exponential factor determines the direction of propagation of the mode: the positive sign corresponds to modes propagating from $-\infty$ in the positive direction of the axis $z, \beta_{j}^{(\alpha)}$ and $\varphi_{j}^{(\alpha)}(x)$ are eigenvalues and eigenfunctions of Sturm-Liouville problems, corresponding the left $(\alpha=1)$ and right $(\alpha=2)$ waveguides accordingly $[4-6,9]$ :

$$
\left\{\begin{array}{l}
\varphi^{\prime \prime}+k_{0}^{2} n_{\alpha}^{2}(x) \varphi=k_{0}^{2} \beta^{2} \varphi, \\
{[\varphi]_{x=0, h_{\alpha}}=\left[\varphi^{\prime}\right]_{x=0, h_{\alpha}}=0,} \\
\varphi\left( \pm R_{x}\right)=0 .
\end{array}\right.
$$

During solution of "the Dirichlet box", we consider the diffraction problem of the guided mode, which propagates in the left waveguide from $-\infty$ in the positive direction of axis $z$. In the case where the left waveguide has the same configuration as the right waveguide, the guided mode passes into the right waveguide without losses. If, however, the configuration of the right waveguide differs from the configuration of the left waveguide, then reflected waveguide modes can appear in the left waveguide - both guided and radiative. In the right waveguide, the transmitted waveguide modes can appear as well, guided and radiative. The field in the left waveguide $(z<0)$ can be represented in the form of a decomposition $[4-6,9]$ :

$$
E_{y}^{(1)}(x, z)=A e^{i k_{0} \beta_{1}^{(1)}} \varphi_{1}^{(1)}(x)+\sum_{j=1}^{\infty} R_{j} e^{-i k_{0} \beta_{j}^{(1)}} \varphi_{j}^{(1)}(x)
$$

where $A$ determines the amplitude of the first waveguide mode, which propagates in the left waveguide from $-\infty$ in the positive direction of the axis $z$.

Amplitude reflection coefficients $R_{j}$ determine the amplitudes of the reflected waveguide modes. The field in the right waveguide $(z>0)$ can be represented in the form of decomposition

$$
E_{y}^{(2)}(x, z)=\sum_{j=1}^{\infty} T_{j} e^{i k_{0} \beta_{j}^{(2)}} \varphi_{j}^{(2)}(x)
$$

where $T_{j}$ determine the amplitudes of the transmitted waveguide modes. At $z=0$ the boundary conditions are satisfied:

$$
\left\{\begin{array}{l}
\left.E_{y}^{(1)}\right|_{z=0}=\left.E_{y}^{(2)}\right|_{z=0}, \\
\left.\frac{\partial E_{y}^{(1)}}{\partial z}\right|_{z=0}=\left.\frac{\partial E_{y}^{(2)}}{\partial z}\right|_{z=0} .
\end{array}\right.
$$

\section{Symbolic solution}

The equation for the Sturm-Liouville problem (10) is considered in three domains where $n^{2}(x)$ remains constant:

$$
\psi_{c}^{\prime \prime}+k_{0}^{2}\left(n_{c}^{2}-\beta^{2}\right) \psi_{c}=0, h<x \leq R_{x},
$$

$$
\begin{gathered}
\psi_{f}^{\prime \prime}+k_{0}^{2}\left(n_{f}^{2}-\beta^{2}\right) \psi_{f}=0,0<x<h, \\
\psi_{s}^{\prime \prime}+k_{0}^{2}\left(n_{s}^{2}-\beta^{2}\right) \psi_{s}=0,-R \leq x<0 .
\end{gathered}
$$

We'll solve the problem for the case (14). The characteristic equation is:

$$
\lambda^{2}+k_{0}^{2}\left(n_{c}^{2}-\beta^{2}\right)=0 .
$$

The solution of this equation depends on the value $\beta$, that we still have to find. It is reduced to three cases:

$$
\left\{\begin{array}{l}
n f>\beta>n s>n c, \\
n f>n s>\beta>n c, \\
n f>n s>n c>\beta
\end{array}\right.
$$

For the first case, we obtain the following values of $\lambda$ for three layers of the waveguide:

$$
\begin{aligned}
& \lambda_{1,2}= \pm i k_{0} \sqrt{\beta^{2}-n_{f}^{2}}, \\
& \lambda_{3,4}= \pm k_{0} \sqrt{\beta^{2}-n_{c}^{2}}, \\
& \lambda_{5,6}= \pm k_{0} \sqrt{\beta^{2}-n_{s}^{2}}
\end{aligned}
$$

which correspond to the following values of the functions $\phi$ :

$$
\begin{gathered}
\varphi_{c}=A_{c} e^{k_{0} \sqrt{\beta^{2}-n_{c}^{2}}(x-h)}+B_{c} e^{-k_{0} \sqrt{\beta^{2}-n_{c}^{2}}(x-h)}, h<x \leq R_{x} \\
\varphi_{f}=A_{f} \sin \left(k_{0} \sqrt{n_{f}^{2}-\beta^{2}} x\right)+B_{f} \cos \left(k_{0} \sqrt{n_{f}^{2}-\beta^{2}} x\right), \\
0<x<h, \\
\varphi_{s}=A_{s} e^{k_{0} \sqrt{\beta^{2}-n_{s}^{2}} x}+B_{s} e^{-k_{0} \sqrt{\beta^{2}-n_{s}^{2} x}},-R_{x} \leq x<0 .
\end{gathered}
$$

For these solutions, we have 6 variables. In order to obtain these variables we need to solve a system of linear algebraic equations, which we compose by substituting (14)-(16) into the boundary conditions of the problem for the corresponding layers. The resulting system of linear equations can be represented in the form of the following equation:

$$
M(\beta) \times \vec{A}=\overrightarrow{0} .
$$

Since the solution $\vec{A}=\overrightarrow{0}$ is trivial for us, we need to find solutions for $|M(\beta)|=0$.

Substituting the eigenvalues into (23) and solving this system by the Gauss method with the choice of the main element, we obtain a set of eigenfunctions for the waveguide.

This search for eigenvalues and eigenfunctions is performed for each of the waveguides. Now we can proceed to solving the diffraction problem at the joint of the two waveguides.

The boundary conditions for this problem determine the continuity of the solution at the joint of two waveguides:

$$
\left\{\begin{array}{l}
\left.E_{y}^{(1)}\right|_{z=0}=\left.E_{y}^{(2)}\right|_{z=0}, \\
\left.\frac{\partial E_{y}^{(1)}}{\partial z}\right|_{z=0}=\left.\frac{\partial E_{y}^{(2)}}{\partial z}\right|_{z=0} .
\end{array}\right.
$$

Substituting formulas (11)-(12) into the boundary conditions (24), we obtain a system of relations for finding the amplitude coefficients of the reflection and transmission modes: 


$$
\left\{\begin{array}{l}
A \varphi_{1}^{(1)}(x)+\sum_{j=1}^{+\infty} R_{j} \varphi_{j}^{(1)}(x)-\sum_{j=1}^{+\infty} T_{j} \varphi_{j}^{(2)}(x)=0, \\
i k_{0} \beta_{n}^{(1)} A \varphi_{1}^{(1)}(x)-i k_{0} \sum_{j=1}^{+\infty} \beta_{j}^{(1)} R_{j} \varphi_{j}^{(1)}(x)- \\
-i k_{0} \sum_{j=1}^{+\infty} \beta_{j}^{(2)} T_{j} \varphi_{j}^{(2)}(x)=0 .
\end{array}\right.
$$

From this infinite system we obtain by truncation a system of size $N 1+N 2$, where $N 1$ is the number of eigenfunctions for the left waveguide, N2 - for the right one. For scalar products, orthogonality conditions are observed in this system:

$$
\left\{\begin{array}{l}
\int_{-R x}^{R x} \varphi_{i}^{(1)} \varphi_{j}^{(1)} d x=0, i \neq j \\
\int_{-R x}^{R x} \varphi_{i}^{(2)} \varphi_{j}^{(2)} d x=0, i \neq j .
\end{array}\right.
$$

This system can be represented as

$$
\left\{\begin{array}{l}
I \times \vec{r}-M_{1} \vec{t}=-A \times \vec{e}_{1}, \\
M_{2} \times \vec{r}-D \vec{t}=-A \times \vec{e}_{2},
\end{array}\right.
$$

where $\vec{r}$ and $\vec{t}$ are vectors of amplitude coefficients $R_{j}$ and $T_{j}, \quad M_{1}=\left[m_{i, j}^{(1)}\right]$, where $m_{i, j}^{(1)}=\int_{-R x}^{R x} \varphi_{j}^{(2)} \varphi_{i}^{(1)} d x$, $M_{2}=\left[m_{i, j}^{(2)}\right], m_{i, j}^{(2)}=i k_{0} \beta_{i}^{(2)} \int_{-R x}^{R x} \varphi_{j}^{(1)} \varphi_{i}^{(2)} d x, I$ is identity matrix of size $N 1 \times N 1, D=\operatorname{diag}\left\{d_{i}\right\}$, where $d_{i}=i k_{0} \beta_{i}^{(2)} \quad, \quad \vec{e}_{1}=\left\{e_{i 1}\right\} \quad, \quad$ where $\quad e_{i 1}=\int_{-R \mathrm{x}}^{R \mathrm{x}} \varphi_{i}^{(1)} \varphi_{i}^{(1)}$, $\vec{e}_{2}=\left\{e_{i 2}\right\}$, where $e_{i 2}=\int_{-R \mathrm{x}}^{R \mathrm{x}} \varphi_{i}^{(1)} \varphi_{i}^{(2)}$.

By solving this system, we obtain the amplitude coefficients of the reflected and transmitted modes. By studying these modes we will be able to understand the efficiency of energy transfer for this case of waveguide joint.

\section{Numerical solution}

Let's solve this problem in one of the examples. We have a joint of two waveguides with heights $h_{1}=1.5 \lambda$ and $h_{2}=\alpha \lambda$, inside the "box" of height $R_{x}=15 \lambda(\lambda=0.55 \mu \mathrm{m})$. They have a common substrate and a cover layer, the refractive indices of which are equal to $n_{c}=1$ and $n_{s}=1.47$. The refractive index of the waveguide layer material of the first waveguide is $n_{f}^{(1)}=1.565$, of the second one it is also $n_{f}^{(2)}=1.565$. One waveguide mode with amplitude coefficient of $A=1$ is running towards the joint.

The solution of this system is calculated in the Maple 17 computer algebra system $[5,6,7,11,12]$. First, we search for eigenvalues and eigenfunctions for each of the waveguides. We consider the numerical solution for the left waveguide. The matrix determinant $M(\beta)$ graph is shown in Fig. 2.

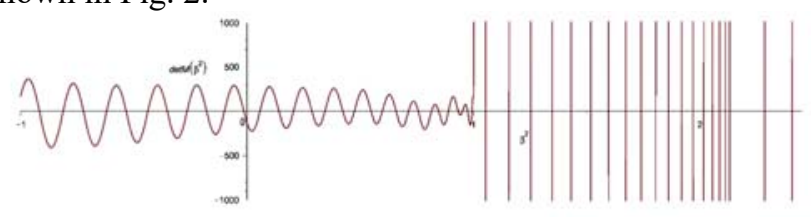

Fig. 2. Graph of determinant of $M(\beta)$.

By solving the equation $\operatorname{det} M(\beta)=0$ we obtain a set of eigenvalues $\beta_{j}^{(1)}$. Substituting the obtained values into the system and solving it by the Gauss method with the search for the main element, we obtain a set of coefficients $A_{c}, B_{c}, A_{s}, B_{s}, A_{f}, B_{f}$ for set of functions $\varphi_{j}^{(1)}$. By substituting them we obtain a set of eigenfunctions. The graphs of the obtained eigenfunctions are shown in Fig. 3-5.

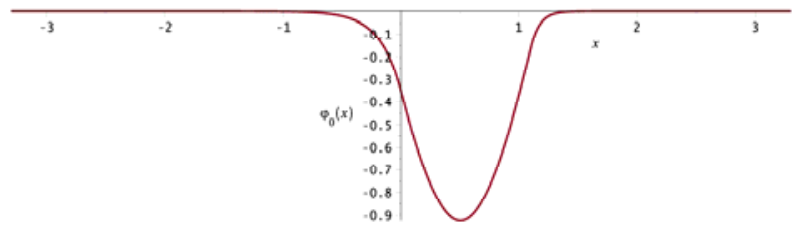

Fig. 3. Graph of $\varphi_{1}^{(1)}(x)$.

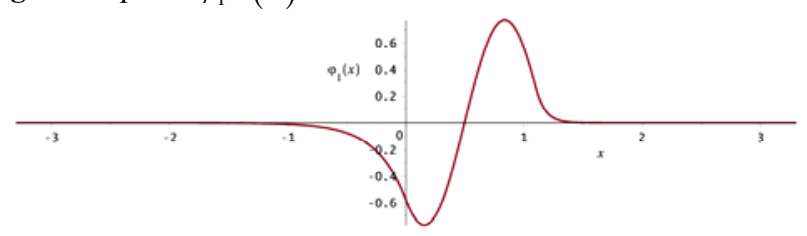

Fig. 4. Graph of $\varphi_{2}^{(1)}(x)$.

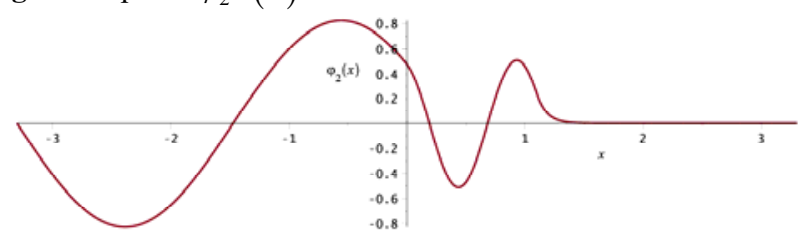

Fig. 5. Graph of $\varphi_{3}^{(1)}(x)$.

By performing the same operations for the second waveguide, we obtain a set of $\beta_{j}^{(2)}$ and $\varphi_{j}^{(2)}$. Now we can proceed to solving the diffraction problem at the joint of two waveguides.

After applying the projection relations of the Galerkin method, we obtained a system (27), the orthogonality conditions of the scalar products are applied for it. To solve the system and find the amplitude coefficients, we use the standard function LinearSolve of the Maple 17 system. The resulting solution vector contains the amplitude coefficients of the reflected and transmitted waveguide modes. 
We will investigate the variation of the transmission coefficients of the first two waveguide modes of the right waveguide as a function of the parameter $\alpha$ - see fig.6.

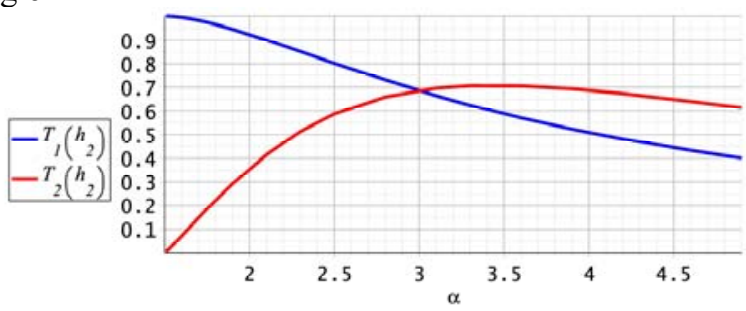

Fig. 6. Transmission coefficients of the first two waveguide modes.

As we can see from the fig.6. for $\alpha=1.5$ - that is, when both waveguides have the same thickness - the transmission coefficient of the first mode is almost equal to 1 , the transmission coefficient of the second mode is almost zero, since almost all of the energy is transferred by the first mode. As the parameter $\alpha$ is increased, the transmission coefficient of the first mode decreases, and the transmission coefficient of the second mode, on the contrary, increases. A further increase in the thickness of the right waveguide leads to the fact that the first mode incident on the waveguide joint excites simultaneously two directional modes, and for $\alpha=3.5$ the second waveguide of the right waveguide mode transfers the main energy.

The accuracy of this solution can be determined by summing up the intensities of the transmitted and reflected modes and comparing them with the intensity of the incident mode:

$$
\left.|| A\right|^{2}-\sum_{j=1}^{N 1}\left|R_{j}\right|^{2}-\sum_{j=1}^{N 2}\left|T_{j}\right|^{2} \mid=\delta
$$

In our case, the difference is $\delta=0.0002$ (much less than $1 \%$ ), that gives satisfactory accuracy of the solution.

\section{Conclusion}

In this paper we described the algorithm for solving the diffraction problem for waveguide modes at the joint of two planar three-layer waveguides. The proposed approach of the "Dirichlet box" makes it possible to approximately analyze the intensity distribution along the guided and radiative modes of the open waveguide for the diffraction problem of waveguide modes at the waveguide joint. We described the method, developed the symbolic-numerical algorithm based on it, and conducted the numerical experiment with the usage of presented algorithm.

The described algorithm makes it possible to study the energy transfer during the propagation of waveguide modes through junctions of different configurations and to numerically estimate the redistribution of energy between modes, which is important in waveguide matching problems.

The publication has been prepared with the support of the "RUDN University Program 5-100".

\section{References}

1. D. Marcuse, Light Transmission Optics, (Van Nostrand Reinhold, 1972).

2. M.J.Adams, An Introduction to Optical Waveguides, (John Wiley \& Sons, New York, Wiley, 1981).

3. T.Tamir, Guided-Wave Optoelectronics, (SpringerVerlag Berlin Heidelberg, 1988).

4. A.G. Sveshnikov, U.S.S.R. Comput. Math. Math. Phys., 3, 1, 170-179, (1963).

5. D.V. Divakov, M.D. Malykh, L.A. Sevastianov, A.L. Sevastianov, E.A. Ayryan, EPJ Web of Conferences, 173, 02007, (2018).

6. D.V. Divakov, L.A. Sevastianov, N.E. Nikolaev, Journal of Physics: Conference Series, 788, 1, 012010, (2017).

7. A.A. Gusev, O. Chuluunbaatar, S.I. Vinitsky, et al., Physics of Atomic Nuclei, 75, 10, 1210-1226, (2012).

8. L.V. Kantorovich, V.I. Krylov, Approximate Methods of Higher Analysis, (New-York, Wiley, 1964).

9. D.V. Divakov, L.A. Sevastianov, N.E. Nikolaev, EPJ Web of Conferences, 108, 02020, (2016).

10. M.D. Malykh, Journal of Mathematical Sciences, 209, 6, 935-952, (2015).

11. D.S. Kulyabov, A.V. Korolkova, L.A. Sevastianov, Journal of Physics: Conference Series, 788, 1, 012025, (2017).

12. L.A. Sevastyanov, A.L. Sevastyanov, A.A. Tyutyunnik, Lecture Notes in Computer Science, 8660, 419-431, (2014). 